\title{
Conocimiento de asma: intervención educativa con la guía GINA 2014 en médicos de primer contacto
}

César Fireth Pozo-Beltrán, ${ }^{1}$ Elsy Maureen Navarrete-Rodríguez, ${ }^{1}$ Roberto Fernández-Soto, ${ }^{1}$ Jazmín Navarro-Munguía, ${ }^{1}$ Margareth Sharon Hall-Mondragón, ${ }^{1}$ Juan José Sienra-Monge ${ }^{2}$ Blanca Estela Del Río-Navarro ${ }^{1}$

\begin{abstract}
Background: Asthma is a public health problem in the world, so updating the guidelines for the diagnosis and treatment of asthma is based primarily on the practice of primary care physicians. Educational interventions are useful for increasing knowledge.

Objective: To compare the level of knowledge of asthma before and after an educational intervention.

Methods: A quasi-experimental prospective study was conducted in general and family practitioners and pediatricians who attended a training workshop on general aspects of asthma and current guidelines for diagnosis and treatment (GINA 2014). A questionnaire consisting of 11 multiple choice questions relating to fundamental aspects of the disease and diagnosis, classification, treatment and management of attacks, was used in two assessments, baseline and post-intervention.

Results: A total of 178 patients participated in the study, with knowledge pre-intervention at 25.5 points and post-intervention at 97.5 points on a scale of 100 , with $p<0.05$.

Conclusion: Educational interventions are inexpensive and effective tools to increase the knowledge of health professionals, and they have an impact on improving patient care.
\end{abstract}

Keywords: Asthma; Medical education; Clinical practice guidelines

Este artículo debe citarse como: Pozo-Beltrán CF, Navarrete-Rodríguez EM, Navarro-Munguía J, Hall-Mondragón MS, Sienra-Monge JJ, Del Río-Navarro BE. Conocimiento de asma: intervención educativa con la guía GINA 2014 en médicos de primer contacto. Rev Alerg Mex. 2016;63(4):358-364

'Secretaría de Salud, Hospital Infantil de México Federico Gómez, Departamento de Alergia e Inmunología Clínica Pediátrica. Ciudad de México, México

'Secretaría de Salud, Hospital Infantil de México Federico Gómez, Subdirección de Pediatría Ambulatoria. Ciudad de México, México
Correspondencia: César Fireth Pozo-Beltrán.

bajaallergy@gmail.com

Recibido: 2016-06-30

Aceptado: 2016-09-21 


\section{Resumen}

Introducción: El asma en un problema de salud pública en el mundo, por ello, la actualización de las guías para el diagnóstico y tratamiento de asma se realiza en función principalmente de la práctica de los médicos de primer contacto. Las intervenciones educativas son útiles para el incremento del conocimiento.

Objetivo: Comparar el nivel de conocimiento acerca de asma antes y después de una intervención educativa.

Métodos: Se realizó un estudio prospectivo cuasiexperimental, en médicos generales, familiares y pediatras que asistieron a un curso-taller relativo a aspectos generales del asma y las guías actuales para su diagnóstico y tratamiento (GINA 2014). Mediante un cuestionario constituido por 11 preguntas de opción múltiple que abordaban aspectos fundamentales de la enfermedad como diagnóstico, clasificación, tratamiento y manejo de exacerbaciones, se realizaron dos evaluaciones, una basal y otra posintervención.

Resultados: Un total de 178 paciente participaron en el estudio, con un conocimiento preintervención de 25.5 puntos y posintervención de 97.5 puntos de una escala de 100 , con una $p<0.05$.

Conclusión: Las intervenciones educativas son maniobras de bajo costo y efectivas que incrementan el conocimiento de los profesionales de la salud y tienen impacto en la mejoría de la atención al paciente.

Palabras clave: Asma; Educación médica; Guías de práctica clínica

\section{Abreviaturas y siglas}

GINA, Global Initiative for Asthma

IMSS, Instituto Mexicano del Seguro Social

ISAAC, International Study of Asthma and Allergies in Childhood
ISSSTE, Instituto de Seguridad y Servicios Sociales de los Trabajadores del Estado NHLBI, National Heart Lung, and Blood Institute OMS, Organización Mundial de la Salud UNAM, Universidad Nacional Autónoma de México

\section{Introducción}

El asma se define como una enfermedad heterogénea caracterizada por inflamación crónica de la vía aérea que provoca síntomas como sibilancias, dificultad respiratoria, opresión torácica y tos, los cuales varían en tiempo e intensidad y se relacionan con limitación variable del flujo aéreo espiratorio. ${ }^{1}$

De acuerdo con la Organización Mundial de la Salud (OMS), actualmente existen 300 millones de personas que padecen asma; anualmente se atribuyen 250000 muertes a esta enfermedad; ${ }^{2,3}$ en Estados Unidos representa la tercera causa de hospitalización en los sujetos menores de 15 años, con un promedio anual de 640000 visitas a urgencias por exacerbaciones, ${ }^{4}$ lo que se traduce en un costo directo de 50.1 billones de dólares e indirecto de 5.9 billones cada año. ${ }^{5}$

En México se ha reportado una prevalencia de $9.5 \%$ y en particular de $5 \%$ en la Ciudad de México, ${ }^{6}$ de acuerdo con el International Study of Asthma and Allergies in Childhood (ISAAC).

El asma es considerado un problema de salud pública que afecta principalmente a los niños y que tiene un impacto negativo en la calidad de vida tanto del paciente como del núcleo familiar. ${ }^{1,7}$ Debido a ese gran impacto, durante años se ha tratado de mejorar el diagnóstico y tratamiento de esta enfermedad mediante la implementación de guías de práctica clínica dirigidas a los médicos de primer contacto, ${ }^{1,8}$ la primera guía en el tema se publicó a mediados de 1980 y desde que se implementó, se observó que coadyuvó en la mejora de las condiciones de diagnóstico y tratamiento de los pacientes con asma.

Actualmente, una de las guías más importantes es primera fue publicada en 1995. Con el transcurso de los años, esta guía ha sido actualizada y se han incluido secciones que hacen énfasis en la importancia de la educación del paciente, la capacitación continua 
y la mejora del entorno; también se han agregado apartados especiales relativos al manejo de los niños menores de 6 años, de las exacerbaciones y de la enfermedad pulmonar obstructiva crónica.

Durante años, en México y en el resto del orbe se han realizado intervenciones educativas encaminadas a mejorar el conocimiento en las guías de práctica clínica por parte del personal médico, tanto de primer contacto como de segundo y tercer nivel, lo que ha permitido realizar una evaluación de la difusión de estos materiales, así como objetivar la importancia de la educación continua del personal de atención de la salud.

En el ámbito internacional se han realizado estudios como el de García Luzardo y colabores, ${ }^{9}$ en el cual se evaluaron los conocimientos de residentes de tercer y cuarto año de la especialidad de pediatría respecto a la Guía Española para el Manejo de Asma (2009). Se aplicó un cuestionario en los principales hospitales con sedes de la especialidad; solo $30 \%$ de los residentes conocía la guía y de esa proporción más de 50\% la utilizaba de forma parcial. Los autores llegaron a la conclusión de que el conocimiento sobre la guía no era bueno.

En 2004, en Puebla, México, Rodríguez Martínez y colaboradores ${ }^{10}$ publicaron una investigación en la que evaluaron el conocimiento en la guía GINA en 1474 médicos de ese estado: 1468 médicos generales y 16 especialistas. Entre julio y septiembre de 1999 se captó a la población de estudio en su consultorio particular o en las sedes de congresos. Se realizó una encuesta con 30 reactivos; la primera parte abordaba el conocimiento relativo al asma y la segunda, al tratamiento. Solo $23 \%$ conocía la guía y $65 \%$ solo identificaba el asma como una enfermedad crónica.

En un estudio de 2004 realizado por Ruiz Espiricueta y colaboradores ${ }^{11}$ en Monterrey, Nuevo León, se seleccionaron 60 médicos de primer nivel que recibieron un curso intensivo sobre asma, diagnóstico y tratamiento con una duración total de 6 horas. Antes del inicio del curso se aplicó un cuestionario de 19 preguntas elaborado por expertos en el tema, así como al terminar el curso. Se identificó que un curso corto de asma basado en conferencias incrementa de manera importante el conocimiento de los médicos de primer nivel de atención y mejora el conocimiento de la guía GINA y su aplicación.

Por su parte, Segura Méndez y colaboradores, ${ }^{12}$ en 2008, describieron el incremento del conocimiento sobre las guías ARIA y GINA 2006 en 69 médicos de primer contacto. La intervención educativa consistió en proporcionar un curso-taller que constaba de 4 módulos con una duración de 40 minutos cada uno; los temas que se abordaron fueron etiología, fisiopatogenia, diagnóstico, espirometría, flujometría, tratamiento y casos clínicos. Se realizó una evaluación pre y postaller con un cuestionario elaborado por expertos en el tema (20 preguntas GINA y 10 ARIA). Dado que hubo una mejoría en la calificación después del curso-taller con una diferencia significativa $(\mathrm{p}<0.05)$, se planteó que una maniobra de bajo costo puede ser efectiva e impactar directamente en la calidad de la atención del paciente con asma.

En el mismo año, Becerril Ángeles y colaboradores ${ }^{13}$ evaluaron el conocimiento en la guía GINA en médicos de diferentes niveles de atención médica del Centro Médico Nacional La Raza. Aplicaron un cuestionario de 19 preguntas a 179 galenos; observaron que los del primer nivel de atención obtuvieron la menor puntuación y que los alergólogos y neumólogos del tercer nivel presentaron mayor conocimiento sobre el tema.

A pesar de los resultados favorables reportados con las maniobras de intervención educativa, poco se ha hecho por perfeccionar estas herramientas; además, durante los últimos años las guías para el diagnóstico y tratamiento del asma han sido modificadas en forma importante, por lo que la capacitación y evaluación debe ser continua.

El objetivo de la investigación que se presenta fue comparar el conocimiento de médicos de primer contacto, antes y después de su participación en talleres y conferencias sobre el diagnóstico y manejo de los pacientes con asma.

\section{Métodos}

Se realizó un estudio prospectivo cuasiexperimental en médicos generales, familiares y pediatras en quienes se evaluó de manera basal el conocimiento que tenían del asma a partir de la guía GINA 2014. Se aplicó un cuestionario de 11 preguntas de opción múltiple, las cuales incluían aspectos fundamentales de la enfermedad como diagnóstico, clasificación, tratamiento y manejo de exacerbaciones; dicho cuestionario fue construido por un panel de 7 alergólogos expertos en la materia, que inicialmente analizaron 25 ítems y que por consenso solo incluyeron 11 por su relevancia. 


\begin{tabular}{|c|c|c|}
\hline \multicolumn{3}{|c|}{$\begin{array}{l}\text { Cuadro 1. Variables sociodemográficas de } 178 \text { médicos de primer } \\
\text { contacto que acudieron a un curso-taller sobre asma }\end{array}$} \\
\hline & Rango & Media $\pm \mathrm{DE}$ \\
\hline Edad (años) & $23-71$ & $47.5 \pm 11.8$ \\
\hline Sexo & $\mathrm{n}$ & $\%$ \\
\hline Femenino & 115 & 64.60 \\
\hline Masculino & 63 & 35.40 \\
\hline $\mathrm{DE}=$ desviación estándar & & \\
\hline
\end{tabular}

El curso-taller se impartió en una sola sesión con una duración de 6 horas. Por medio de conferencias se abordaron aspectos generales del asma y las guías actuales para su diagnóstico y tratamiento (GINA 2014). Las conferencias fueron impartidas por expertos certificados por el Consejo Nacional de Inmunología Clínica y Alergia de México. Al terminar la parte teórica se inició un taller de casos clínicos, de espirometría y uso de inhaladores, después de lo cual se aplicó de nuevo el cuestionario.

Se revisaron y calificaron los instrumentos que se aplicaron para obtener evaluaciones individuales y generales; se realizó análisis univariado de las variables demográficas utilizando medidas de tendencia central y de dispersión, mientras que la comparación de grupos se realizó con $\chi^{2}$. Todas las estimaciones estadísticas se efectuaron con el programa estadístico SPSS versión 20.0.

\section{Resultados}

Se incluyeron 178 médicos de primer contacto, con una edad media \pm desviación estándar de $47.5 \pm 11.8$ años; $64.6 \%$ era del sexo femenino (IC 95\%, 0.530.74 ) y $35.4 \%$ del masculino (IC $95 \%, 0.25-0.46$ )

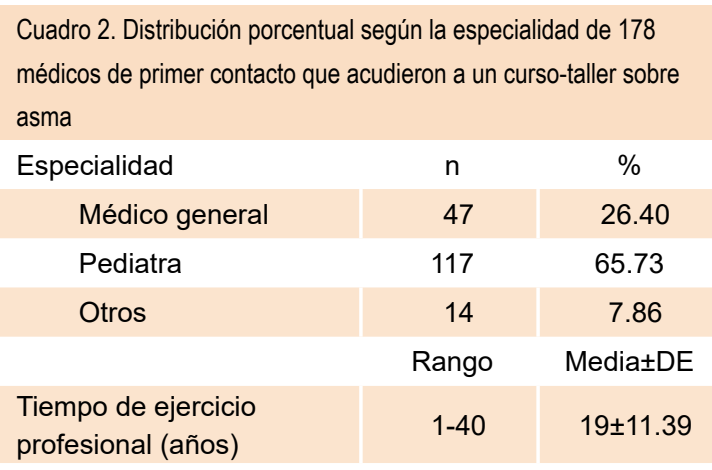

(Cuadro 1). En cuanto a la profesión de los médicos participantes, $65.73 \%$ (IC 95\% 0.54-0.75) eran pediatras, con una media de años de ejercicio profesio-

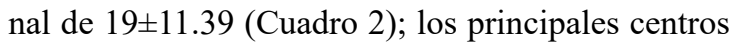
de donde acudían dichos médicos correspondían al sector privado, $56.17 \%$ (IC 95\% 0.45-0.67); el resto ejercía en el sector público (Cuadro 3).

Antes del curso-taller se aplicó el cuestionario, con el cual se obtuvo una puntuación general de los asistentes de 25.5 de una escala de 100; después de la intervención educativa se obtuvo 97.5.

La mejoría del conocimiento después del cursotaller fue estadísticamente significativa $(\mathrm{p}<0.05)$ (Cuadro 4 y Figura 1).

Por otra parte, en cuanto al uso de una aerocámara para el tratamiento de asma solo $40 \%$ lo prescribía en la consulta; en cuanto al uso correcto de los diferentes dispositivos para el tratamiento del asma solo $10 \%$ de los participantes conocía la técnica correcta de aplicación, con los cuidados pre y postratatamiento.

\section{Discusión}

Las guías de práctica clínica sobre asma se actualizan constantemente y la información que proporcionan sobre diagnóstico, tratamiento y manejo de exacerbaciones está sustentada en las mejores evidencias, además, cada apartado aborda las peculiaridades según los diferentes grupos de edad.

Las guías están dirigidas principalmente a los médicos de primer contacto, a quienes aporta información relevante para mejorar la atención del paciente y evitar complicaciones de la enfermedad.

Dado que la capacitación continua del personal de salud es de gran importancia, el objetivo de la investigación fue evaluar el nivel de conocimiento de la guía de asma en médicos de primer contacto y conforme a eso se impartió un curso-taller sobre la información proporcionada en la guía GINA 2014. $\mathrm{Al}$ igual que lo observado en otras investigaciones, el conocimiento y la aplicación en la práctica clínica cotidiana de la guía fueron muy bajos, ${ }^{9,10}$ aun cuando se trata de un documento de acceso libre y gratuito.

Las intervenciones educativas son herramientas que pueden mejorar el conocimiento del personal de salud, de ahí que autores nacionales e internaciones se preocupen por proponer diversas estrategias.

Después de la intervención educativa con el curso-taller, hubo un incremento estadísticamente 
significativo del conocimieno, al igual que en otras investigaciones que han demostrado que esta herramienta es de bajo costo y efectiva, y que su impacto se refleja en el diagnóstico temprano, tratamiento oportuno y disminución de las complicaciones. ${ }^{11-13}$

Dado que los resultados positivos se evaluaron de manera inmediata, no tenemos la posibilidad de determinar por cuánto tiempo perdurarán; de los médicos evaluados, $28.1 \%$ ya había tomado anteriormente el curso. De ahí la necesidad de diseñar herramientas educativas que constantemente refuercen el conocimiento aprendido.

Además de la preparación en cuanto al conocimiento en la guía, se proporcionó un taller sobre el uso de dispositivos para el tratamiento del asma. El médico, además de otorgar un servicio de salud, debe orientar al paciente en diferentes aspectos, uno de ellos es la aplicación del medicamento, que tiene un impacto importante en el control de la enfermedad. Antes de la intervención educativa se observó pobre conocimiento acerca de los dispositivos para
Cuadro 3. Distribución porcentual según institución de procedencia de 178 médicos de primer contacto que acudieron a un curso-taller sobre asma

Institución

Sector privado

Sector público

Secretaría de Salud

IMSS

ISSSTE

UNAM

$\mathrm{n}$

$\%$

100
78

78

56.17

IMSS, Instituto Mexicano del Seguro Social; ISSSTE, Instituto de Seguridad y Servicios Sociales de los Trabajadores del Estado; UNAM, Universidad Nacional Autónoma de México

el tratamiento; después del curso-taller, el conocimiento aumentó en forma importante, lo cual indudablemente se reflejará en la calidad de vida del paciente.

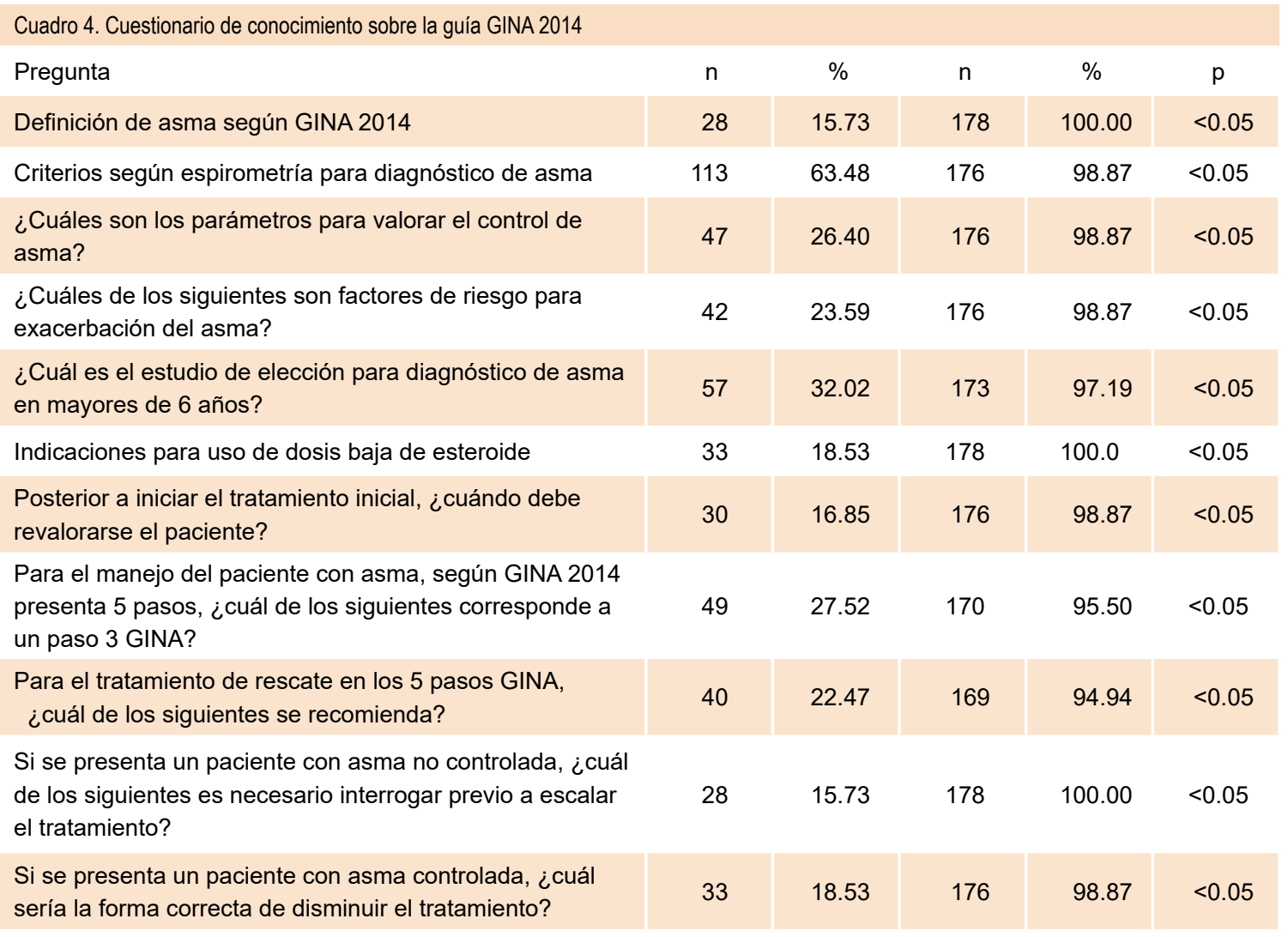




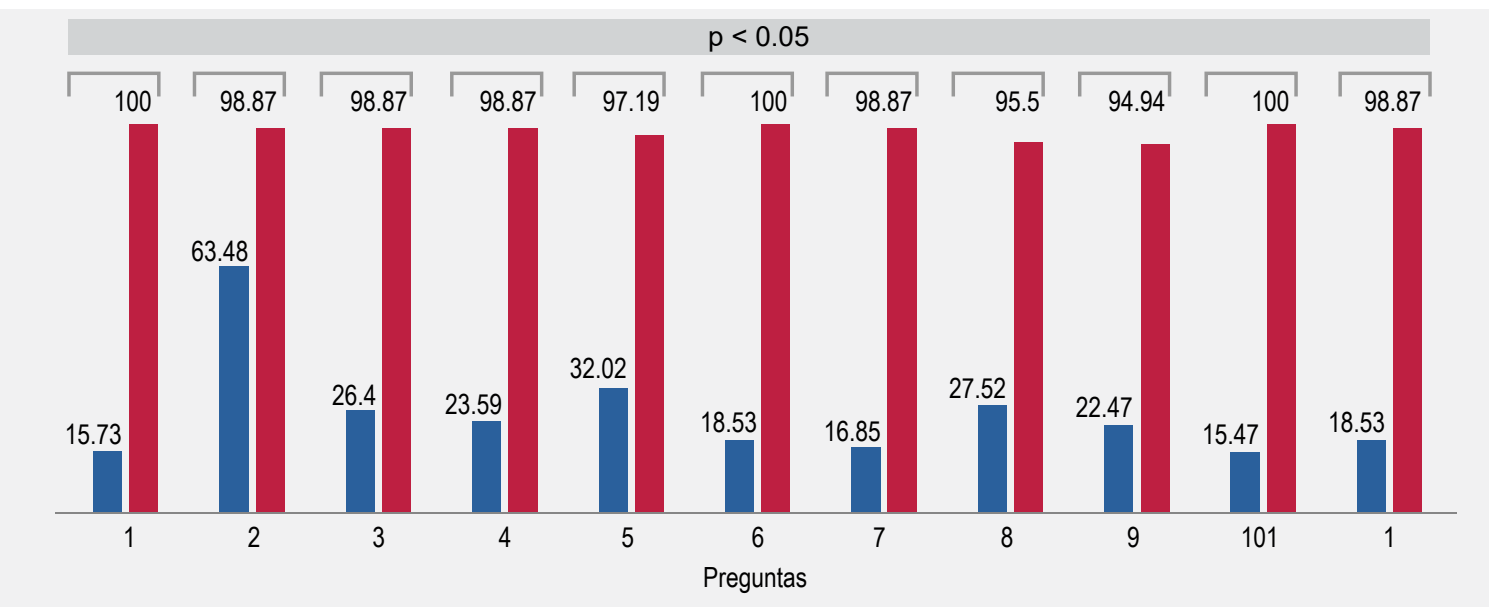

Figura 1. Distribución porcentual según respuesta correcta pre y posintervención educativa en el conocimiento de asma.

\section{Conclusiones}

Los resultados de nuestra investigación concuerdan con los de estudios internacionales respecto a la mejoría del conocimiento en asma después de una intervención educativa. La mejoría en los conocimientos, la adquisición de habilidades necesarias y el cambio en la actitud y los comportamientos de los médicos de primer contacto es indispensable para que orienten a los pacientes, familiares y cuidadores, con lo que se facilita el apego al tratamiento y permite un mejor manejo de los síntomas y de la enfermedad.

Así mismo, el nivel bajo de conocimientos del médico se relaciona con pobre control de la enfer- medad ya que puede ocasionar que se clasifique erróneamente al paciente, se otorgue un tratamiento no óptimo y, en consecuencia, un mal control de la enfermedad. La intervención educativa del personal de salud sigue siendo un pilar importante en el manejo y tratamiento del asma.

Para finalizar, parece necesario reflexionar sobre la importancia de los programas informativos que aseguren el adecuado conocimiento de enfermedades como el asma, cuya prevalencia e impacto así lo requieren. Vale la pena adoptar estrategias eficaces para la difusión de las guías de práctica clínica sobre asma entre los médicos del primer contacto.

\section{Referencias}

1. Global Initiative for Asthma. [Sitio web]. The Global Strategy for Asthma Management and Prevention, 2014. [Actualización: 2016 GINA Report, Global Strategy for Asthma Management and Prevention]. Disponible en: http://ginasthma.org/2016-gina-report-global-strategy-for-asthma-management-and-prevention/

2. World Allergy Organization. [Sitio web]. WAO White Book on Allergy, 2011. Disponible en: http://www. worldallergy.org/UserFiles/file/WAO-White-Book-on-Allergy_web.pdf

3. World Health Organization. [Sitio web]. Global surveillance, prevention and control of chronic respiratory diseases. A comprehensive approach. Geneva: WHO; 2007. Disponible en: http://www.who.int/gard/ publications/GARD\%20Book\%202007.pdf

4. Centers for Disease Control and Prevention. CDC Wonder [Sitio web]. Compressed Mortality File Archives 1999-2013. Disponible en: https://wonder.cdc.gov/mortArchives.html

5. Barnett SB, Nurmagambetov TA. Costs of asthma in the Unites States: 2002-2007. J Allergy Clin Immunol. 2011;127(1):145-152. doi: 10.1016/j.jaci.2010.10.0

6. Del Río-Navarro B, Del M. Asthma prevalence in children living in North Mexico City and a comparison with other Latin American cities and world regions. Allergy Asthma Proc. 2006;27(4):334-340. 
Pozo-Beltrán CF et al. Intervención educativa con la guía GINA en médicos de primer contacto

7. American Lung Association. Trends in asthma morbidity and mortality. Chicago, IL, US: American Lung Association; 2012. Disponible en: http://www.lung.org/assets/documents/research/asthma-trend-report. pdf

8. Díaz-Vázquez CA. Asma: protocolos, guías y consensos ¿con cuál quedarse? An Pediatr (Barc). 2006; 64(6):511-514. Disponible en: http://www.analesdepediatria.org/es/asma-protocolos-guias-consensos-con/ articulo/S1695403306701163/

9. García-Luzardo MR, Aguilar-Fernández AJ, Rivero-Rodríguez SM. Conocimiento de los residentes de tercer y cuarto año de pediatría de la Guía Española para el Manejo del Asma 2009 (GEMA 2009). Acta Pediatr Esp. 2011;69(6):267-275.

10. Rodríguez Martínez JI, Bazán-Riverón GE, Paredes-Rivera MP, Osorio-Guzmán M, Caso-Marasco A, Sandoval-Navarrete J. Evaluación del conocimiento de GINA en médicos generales y especialistas del estado de Puebla (México). Alerg Asma Inmunol Pediatr. 2004;13(3):94-98. Disponible en: http://www. medigraphic.com/pdfs/alergia/al-2004/al043a.pdf

11. Ruiz-Espiricueta JE, González-Díaz SN, Galindo-Rodríguez G. Evaluación de un curso de educación en asma para médicos del primer nivel de atención. Rev Alerg Mex. 2005;52(2):83-89. Disponible en: http:// www.medigraphic.com/pdfs/revalemex/ram-2008/ram085d.pdf

12. Segura-Méndez NH, Velázquez AC, Rivero HL, Hernández VL, Espinola RG, Mondragón GR. Incremento del conocimiento de las guías ARIA y GINA 2006 para médicos generales mediante una intervención educativa. Rev Alerg Mex. 2008;55(5):201-205. Disponible en http://www.medigraphic. com/pdfs/revalemex/ram-2008/ram085d.pdf

13. Becerril-Ángeles M, León-Alvarado F, Ángeles-Garay U. Evaluación del conocimiento de la GINA en médicos de diferentes niveles de atención. Rev Alerg Mex. 2007;54(2):29-33. Disponible en: http://www. medigraphic.com/pdfs/revalemex/ram-2007/ram072a.pdf 